Portland State University

PDXScholar

$11-1-2002$

\title{
Nonlocal Electrodynamic Modeling of Fluorescence Characteristics for Molecules in a Spherical Cavity
}

\author{
Pui T. Leung \\ Portland State University, hopl@pdx.edu \\ M. H. Hider \\ Portland State University
}

Follow this and additional works at: https://pdxscholar.library.pdx.edu/phy_fac

Part of the Physics Commons

Let us know how access to this document benefits you.

\section{Citation Details}

Hider, M. H., \& Leung, P. T. (2002). Nonlocal electrodynamic modeling of fluorescence characteristics for molecules in a spherical cavity. Physical Review B (Condensed Matter And Materials Physics), 66(19), 195106-1-7.

This Article is brought to you for free and open access. It has been accepted for inclusion in Physics Faculty Publications and Presentations by an authorized administrator of PDXScholar. Please contact us if we can make this document more accessible: pdxscholar@pdx.edu. 


\title{
Nonlocal electrodynamic modeling of fluorescence characteristics for molecules in a spherical cavity
}

\begin{abstract}
M. H. Hider and P. T. Leung*
Department of Physics, Portland State University, P.O. Box 751 Portland, Oregon 97207-0751

(Received 12 June 2002; revised manuscript received 6 September 2002; published 13 November 2002)

The emission characteristics for molecules in a spherical metallic microcavity are computed using a nonlocal electrodynamic model, based on a theory previously published by Fuchs and Claro [Phys. Rev. B 35, 3722 (1987)] for the multipole polarizability of a sphere. Both radially and tangentially oriented molecules at arbitrary locations inside the cavity are considered, and the results are compared with those from both the local response theory and those for molecules outside a spherical particle. The issue of reciprocity of the solutions for each of the sphere and cavity cases, respectively, is examined in the light of the nonlocal effects. It is observed that for emission frequencies below the surface plasmon frequency of the cavity material, the nonlocal effects in general lead to less surface-induced modifications of the molecular properties, similar to the situation for a spherical particle. However, the reciprocity nature between the solutions for the sphere and cavity disappears in the presence of nonlocal effects.
\end{abstract}

DOI: $10.1103 /$ PhysRevB.66.195106

PACS number(s): 41.20.-q, 42.50.Ct, 42.60.Da, 33.70.Jg

\section{INTRODUCTION}

The emission characteristics from molecules confined in a microcavity have been intensively studied in the last two decades in a variety of different contexts such as cavity quantum electrodynamics (CQED) (Ref. 1) and semiconducting microcavities. ${ }^{2}$ Due to the modification of the photonic density of states in the cavity, ${ }^{3}$ subtle QED effects can be manipulated and studied, and various molecular emission properties can be controlled by adjusting the cavity parameters. Aside from emissions from individual molecules, cooperative emission and energy transfer among a group of the molecular dipoles in the cavity can also be dramatically modified by varying these parameters. ${ }^{4}$ Potential applications by achieving such control of molecular emission and interaction properties in a cavity range over a diversity of fields, from photochemistry to optoelectronics. ${ }^{5}$

Among the different geometries, the spherical cavity is the one which has been studied very frequently by both theorists and experimenters. Because of the simple geometry, the theoretical solution of a dipole (or a two-level system) in such a cavity can be obtained with high accuracy and analyticity, in both classical and quantum mechanical approaches. $^{6-12}$ In addition, experimental studies on such systems can also be achieved via spectroscopic monitoring of dissolved ions and dyes in liquid microdroplets. ${ }^{13-15} \mathrm{Be}-$ sides spectroscopy, the solution to this problem can also provide for a useful solvent model in physical chemistry. ${ }^{16}$

In our present study, we will establish a model which allows us to calculate the modified emission frequency and the decay rate for molecules in a spherical cavity using nonlocal electrodynamics. Although quantum mechanical models for this problem have been considered in the literature, the classical phenomenological (CP) model often provides simple approach which can account for many experiments with sufficient accuracy. ${ }^{3,17}$ In particular, detailed parameters of the emitting molecule such as the relative location and the transition dipole orientation in the cavity can easily be incor- porated in the $\mathrm{CP}$ approach. In a previous experiment by Barnes et al., ${ }^{14}$ the CP model was found to lead to a discrepancy for droplets with dimensions much greater than the emission wavelengths of the molecules, which are dissolved inside the droplets. A subsequent theoretical investigation based on quantum theory in the strong-coupling condition between the dipole and cavity was reported to be able to account for the discrepancy observed. ${ }^{9}$ However, as in most CP models, ${ }^{6-8,10-12}$ nonlocal effects were ignored in the model applied by Barnes et al., and these effects can be significant, especially for molecules near the surface of the microdroplets. These nonlocal effects refer to the wave vector dependence of the dielectric response of the cavity or droplet. In our work below, we shall study these effects in the CP model and shall limit our formulation to cavities with dimensions small compared to the emission wavelengths, so that retardation effects can be ignored. Under this limitation, the dipole-cavity interaction can be approximated using electrostatic solutions and the nonlocal effects can be introduced in a relatively simple way. It is of interest to note that fluorescence of molecules in these "nanobubbles" has been studied intensively in several recent works. ${ }^{8,11,12}$ Moreover, it appears that none of these has considered nonlocal effects, which can be of significance as demonstrated below.

\section{THEORETICAL MODEL}

The nonlocal theory for the electromagnetic interaction between a charge or dipole and a spherical particle or cavity in the $\mathrm{CP}$ approach has been intensively studied in the literature. This includes, for example, the continuum solvent model of Basilevsky and Parsons, ${ }^{16}$ the generalized susceptibility (propagator) method of Labani and co-workers, ${ }^{18}$ and the nonlocal theory for the multipolar polarizability of spheres introduced by Fuchs and Claro (FC). ${ }^{19}$ Previously, we have applied the FC model to study the emission characteristics of molecules near a spherical particle. ${ }^{20}$ In the following, we shall extend the FC model to the case of a dipole inside a spherical cavity (of radius $a$ ), accounting for the 


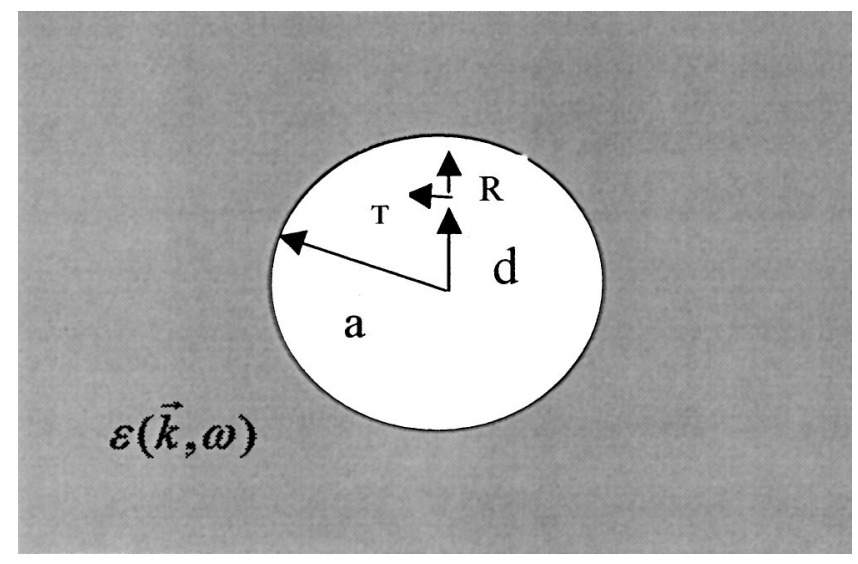

FIG. 1. Geometry of the dipole-cavity problem.

radial and tangential orientations of the transition dipole moment of the molecule. To be specific, we shall assume the inside of the cavity to be vacuum surrounded by a metallic medium.

Consider a spherical boundary at $r=a$. The main idea of the FC model is to assume a fictitious continuation of the medium (with a nonlocal dielectric response) beyond the geometrical boundary $(r=a)$. By introducing an additional boundary condition $(\mathrm{ABC})$ to require the radial components of the displacement vectors $\left(D_{r}\right)$ to be discontinuous (corresponding to the presence of a fictitious external surface charge at $r=a$ ), the electric field can then be smoothly extended across the geometrical boundary, thus accounting for the nonlocal dielectric response of the medium.

Without loss of generality, we consider a dipole with an arbitrary location on the $z$ axis. For a dipole $(\vec{\mu})$ at $(d, 0,0)$ oriented radially (along the $z$ axis) in a (vacuum) spherical cavity (Fig. 1), the electrostatic potentials can be obtained by following the FC theory ${ }^{19}$ as follows:

$$
\begin{gathered}
\Phi(d<r<a)=\sum_{l}\left(A_{l} r^{l}+\mu l \frac{d^{l-1}}{r^{l+1}}\right) P_{l}(\cos \theta), \\
\Phi(r>a)=\sum_{l} \delta a^{2} F_{l}(r) P_{l}(\cos \theta), \\
\Phi_{D}(r>a)=\sum_{l} \delta a^{2} \frac{1}{2 l+1} \frac{a^{l}}{r^{l+1}} P_{l}(\cos \theta),
\end{gathered}
$$

where

$$
F_{l}(r)=\frac{2}{\pi} \int_{0}^{\infty} \frac{j_{l}(k a) j_{l}(k r)}{\varepsilon(k, \omega)} d k .
$$

In the above equations, $\delta$ is the discontinuity of $D_{r}$ into the fictitious medium, $\varepsilon(k, \omega)$ is the nonlocal dielectric function of the isotropic medium, $P_{l}$ is the Legendre polynomial, and $j_{l}$ is the spherical Bessel function. Note that the two potentials in the medium give the electric and displacement fields, via $\mathbf{E}=-\nabla \Phi$ and $\mathbf{D}=-\nabla \Phi_{D}$, respectively. The corresponding results for a tangential dipole at $(d, 0,0)$ oriented along the $x$ direction can be obtained as

$$
\begin{gathered}
\Phi(d<r<a)=\sum_{l}\left(B_{l} r^{l}-\mu \frac{d^{l-1}}{r^{l+1}}\right) P_{l}^{1}(\cos \theta) \cos \varphi \\
\Phi(r>a)=\sum_{l} \delta a^{2} F_{l}(r) P_{l}^{1}(\cos \theta) \cos \varphi \\
\Phi_{D}(r>a)=\sum_{l} \delta a^{2} \frac{1}{2 l+1} \frac{a^{l}}{r^{l+1}} P_{l}^{1}(\cos \theta) \cos \varphi
\end{gathered}
$$

where $P_{l}^{1}$ is the associated Legendre function.

Following FC, by matching the values of both $\Phi$ and $D_{r}$ (with $D_{r}=-\nabla_{r} \Phi_{D}$ for $r>a$ ) at the geometrical boundary $r$ $=a$, one obtains the coefficients $A_{l}$ in Eq. (1) and $B_{l}$ in Eq. (5) as follows:

$$
\begin{aligned}
& A_{l}=\frac{\mu d^{l-1}}{a^{2 l+1}} l \Delta_{l}^{\mathrm{NL}}, \\
& B_{l}=\frac{\mu d^{l-1}}{a^{2 l+1}} \Delta_{l}^{\mathrm{NL}},
\end{aligned}
$$

where $\Delta_{l}^{\mathrm{NL}}$ is defined as the nonlocal reflection coefficient and is given by

$$
\Delta_{l}^{\mathrm{NL}}=-\frac{\left(\xi_{l}-1\right)(l+1)}{\xi_{l}(l+1)+l},
$$

with $\xi_{l}(\omega)=\left[(2 l+1) a F_{l}(a)\right]^{-1}$ being the "effective dielectric function" obtained through the introduction of a nonlocal response $\varepsilon(k, \omega)$ in Eq. (4) for $F_{l}$. Note that had one worked in the local response theory, one would have obtained very similar results as in Eqs. (1)-(9) except that $\xi_{l}$ in Eq. (10) will be replaced by $\varepsilon(\omega)$, the local dielectric function of the medium. To apply the above results to study the emission characteristics of a molecular dipole in the spherical cavity, we resort to the $\mathrm{CP}$ model $^{17}$ which gives the reduced molecular decay rate and the frequency shifts in the form

$$
\begin{aligned}
& \frac{\gamma}{\gamma_{0}}=1+\frac{3 q}{2 k^{3}} \operatorname{Im}(G), \\
& \frac{\Delta \omega}{\gamma_{0}}=-\frac{3 q}{4 k^{3}} \operatorname{Re}(G),
\end{aligned}
$$

where $q$ is the intrinsic quantum yield of the molecule, $k$ the emission wave vector, and the quantities are normalized with respect to the free decay rate of the molecule. The function $G$ in Eqs. (11) and (12) is defined as the field from the cavity acted on the molecule per unit dipole moment and can be calculated from Eqs. (1) and (5) with the results in Eqs. (8)-(10). The detailed results for $G$ for the two orthogonal dipole orientations are summarized in Table I. For the special case of a "centered dipole," only the $l=1$ term survives in both Eqs. (1) and (5) in the limit $d \rightarrow 0$ and they yield identical results when applied to Eqs. (11) and (12). For comparison and for a complete reference, we have also given the results for the case of a dipole outside a spherical particle and the corresponding results in the local response theory in the same table. 
TABLE I. Summary of results

\begin{tabular}{|c|c|c|c|}
\hline $\begin{array}{l}\text { Dipole } \\
\text { orientation }\end{array}$ & $\begin{array}{l}\text { Geometry (of } \\
\text { environment) }\end{array}$ & Local response theory & Nonlocal response theory ${ }^{a}$ \\
\hline \multirow[t]{4}{*}{ Radial } & \multirow[t]{2}{*}{ Sphere } & $G_{\perp}=-\sum_{l} \frac{(l+1)^{2}}{r_{d}^{2(l+2)}} \Delta_{l}^{\mathrm{sph}} a^{2 l+1}$ & $G_{\perp}=-\sum_{l} \frac{(l+1)^{2}}{r_{d}^{2(l+2)}} \Delta_{l}^{\mathrm{NL}-\mathrm{sph}} a^{2 l+1}$ \\
\hline & & $\Delta_{l}^{\mathrm{sph}}=-\frac{l(\varepsilon-1)}{l(\varepsilon+1)+1}$ & $\Delta_{l}^{\mathrm{NL}-\mathrm{sph}}=-\frac{l\left(\xi_{l}-1\right)}{l\left(\xi_{l}+1\right)+1}$ \\
\hline & \multirow[t]{2}{*}{ Cavity } & $G_{\perp}=-\sum_{l} l^{2} r_{d}^{2(l-1)} \frac{\Delta_{l}^{\mathrm{cav}}}{a^{2 l+1}}$ & $G_{\perp}=-\sum_{l} l^{2} r_{d}^{2(l-1)} \frac{\Delta_{l}^{\mathrm{NL}-\mathrm{cav}}}{a^{2 l+1}}$ \\
\hline & & $\Delta_{l}^{\mathrm{cav}}=-\frac{(l+1)(\varepsilon-1)}{l(\varepsilon+1)+\varepsilon}$ & $\Delta_{l}^{\mathrm{NL}-\mathrm{cav}}=-\frac{(l+1)\left(\xi_{l}-1\right)}{l\left(\xi_{l}+1\right)+\xi_{l}}$ \\
\hline \multirow[t]{4}{*}{ Tangential } & \multirow[t]{2}{*}{ Sphere } & $G_{\|}=-\sum_{l} \frac{l(l+1)}{2 r_{d}^{2(l+2)}} \Delta_{l}^{\mathrm{sph}} a^{2 l+1}$ & $G_{\|}=-\sum_{l} \frac{l(l+1)}{2 r_{d}^{2(l+2)}} \Delta_{l}^{\mathrm{NL}-\mathrm{sph}} a^{2 l+1}$ \\
\hline & & $\Delta_{l}^{\mathrm{sph}}=-\frac{l(\varepsilon-1)}{l(\varepsilon+1)+1}$ & $\Delta_{l}^{\mathrm{NL}-\mathrm{sph}}=-\frac{l\left(\xi_{l}-1\right)}{l\left(\xi_{l}+1\right)+1}$ \\
\hline & \multirow[t]{2}{*}{ Cavity } & $G_{\|}=-\sum_{l} \frac{l(l+1)}{2} \frac{r_{d}^{2(l-1)}}{a^{2 l+1}} \Delta_{l}^{\mathrm{cav}}$ & $G_{\|}=-\sum_{l} \frac{l(l+1)}{2} r_{d}^{2(l-1)} \frac{\Delta_{l}^{\mathrm{NL}-\mathrm{cav}}}{a^{2 l+1}}$ \\
\hline & & $\Delta_{l}^{\mathrm{cav}}=-\frac{(l+1)(\varepsilon-1)}{l(\varepsilon+1)+\varepsilon}$ & $\Delta_{l}^{\mathrm{NL}-\mathrm{cav}}=-\frac{(l+1)\left(\xi_{l}-1\right)}{l\left(\xi_{l}+1\right)+\xi_{l}}$ \\
\hline
\end{tabular}

"The "effective dielectric function" in the nonlocal response theory is defined according to the FC theory (Ref. 19) as

$$
\xi_{l}=\frac{1}{(2 l+l) a F_{l}}=\frac{1}{(2 / \pi)(2 l+1) a \int_{0}^{\infty}\left[j_{l}^{2}(k a) / \varepsilon(k, \omega)\right] d k}
$$

Note that $r_{d}$ is the distance measured from the center of the sphere or cavity to the position of the dipole.

It is clear from Table I that in this approach, all the results for the nonlocal response theory can be obtained from the corresponding results in the local theory by replacing the dielectric function by the "effective dielectric function" $\xi_{l}$ in the FC theory. ${ }^{19}$ We have to remark that the results obtained in our present approach are very similar to those obtained previously by the "propagator method," 18 except that these latter results were expressed in terms of discrete sums over the wave number $k$, which required more involved numerical evaluation. In addition, the previous application of the results obtained in Ref. 18 was limited to the calculation of the dipole-cavity interaction energy and has not been applied to the study of molecular fluorescence, which requires the calculation of also the field strength acting on the dipole. ${ }^{21}$ Incidentally, we note that the method of "reciprocity" 22 which enables one to transform easily between the sphere and cavity results stops working in the nonlocal case, due to the $l$ dependence of the function $\xi_{l}$. This point can be illustrated more explicitly by using a specific model for the nonlocal dielectric function $\varepsilon(k, \omega)$ such as the hydrodynamic model, as shown in the following section.

\section{NUMERICAL RESULTS}

For illustrative purposes, we have computed Eqs. (11) and (12) according to the hydrodynamic model for the nonlocal dielectric response:

$$
\varepsilon(k, \omega)=1-\frac{\omega_{p}^{2}}{\omega(\omega+i \Gamma)-\beta^{2} k^{2}},
$$

where $\omega_{p}$ is the bulk plasmon frequency, $\Gamma$ the damping constant, and $\beta^{2}=\frac{3}{5} v_{F}^{2}, v_{F}$ being the Fermi velocity of the metallic medium. ${ }^{19,20}$ The local result (Drude model) corresponds to setting $\beta=0$ in Eq. (13). Using Eq. (13), the functions $F_{l}$ and hence $\xi_{l}$ and $\Delta_{l}$ can be evaluated in analytical form in terms of the modified Bessel functions $I_{l}$ and $K_{l} .{ }^{19}$ Since upon the transformation $l \rightarrow-(l+1)$ the functions $I_{l}$ and $K_{l}$ do not remain invariant, we see that this reciprocity transformation between the sphere and cavity results for the local case, as obvious from the results in Table I, breaks down in the presence of nonlocal effects.

In our numerical illustrations, we have assumed a vacuum cavity in silver. The numerical constants needed in Eq. (13) for silver are given as follows: ${ }^{20} \omega_{p}=1.36 \times 10^{16} \mathrm{~s}^{-1}, \Gamma$ $=2.56 \times 10^{13} \mathrm{~s}^{-1}$, and $v_{F}=1.40 \times 10^{8} \mathrm{~cm} \mathrm{~s}^{-1}$. Figure 2 shows the computed normalized decay rates (in logarithmic values) as a function of the molecule position at a distance measured from the center of the cavity for a fixed emission frequency at $\omega=0.7 \omega_{s p}$. The diameter of the cavity is fixed at $10 \mathrm{~nm}$. It is seen that while the nonlocal effects in general lead to smaller surface-induced effects, they are particularly significant for molecules close to the cavity wall ( $d$ $=5 \mathrm{~nm}$ ). In addition, while the tangential dipoles experience 


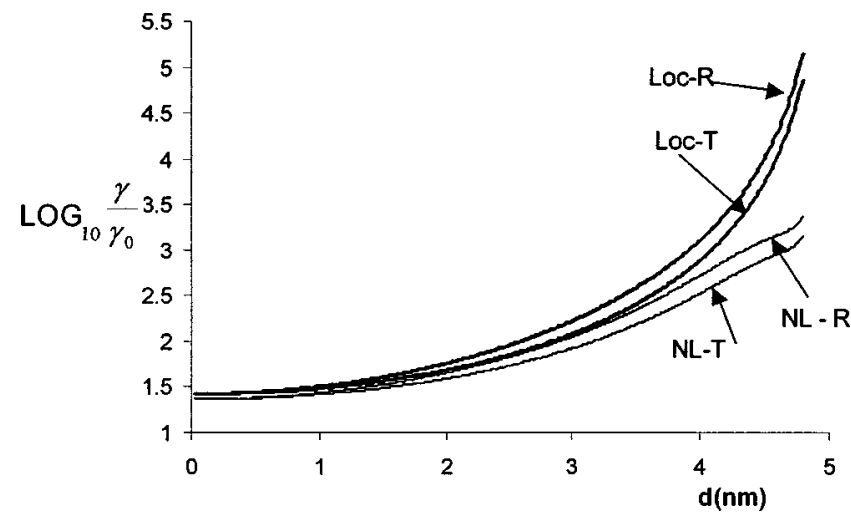

FIG. 2. Normalized decay rate as a function of the position of the molecule at emission frequency (normalized to the surface plasmon frequency) fixed at 0.7 . Distance is measured from the center of the Ag cavity with diameter $D=10 \mathrm{~nm}$. Note that logarithmic values are used for the $y$ axis.

a relatively smaller decay value due to the opposite orientation of the image dipoles, ${ }^{7}$ the results for both orientations merge at the center of the cavity $(d=0)$ as expected. Figure 3 shows the corresponding behavior of the cavity-induced frequency shift (in logarithmic values) in which redshifts are obtained for an emission frequency below the surface plasmon frequency. It is of interest to note that nonlocal effects can lead to a suppression of the surface-induced shifts by an order of magnitude for molecules close to the cavity wall. Next, in Figs. 4 and 5, we show the spectral plot of the decay rates (in logarithmic values) and frequency shifts versus the emission frequency of the molecule. It is noted that while the multipole resonance structures are more pronounced in the nonlocal theory as was observed before in the case of a

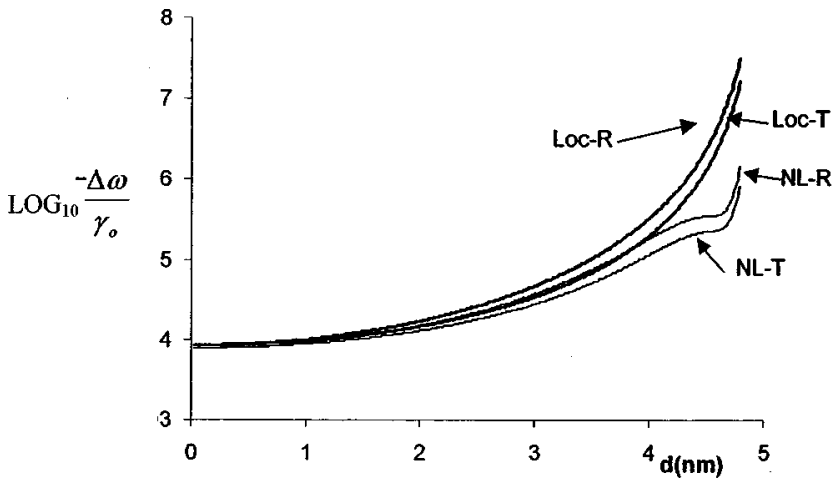

FIG. 3. Frequency shift (normalized to the free decay rate) as a function of distance. The details are the same as those in Fig. 2.

spherical particle, ${ }^{20}$ all the resonance frequencies are blueshifted in the case of a cavity. This can be understood simply by comparing the dipolar surface plasmon resonance of a spherical particle with that of a spherical cavity. While the resonance condition for the sphere in the local theory is $\varepsilon(\omega)+2=0$, that for the cavity can be obtained via a "reciprocity transformation" $\varepsilon \rightarrow 1 / \varepsilon$ (Ref. 23 ) as $1+2 \varepsilon(\omega)=0$. Thus, using the ideal undamped Drude model, the former predicts a resonance frequency of $\omega_{s p}=\omega_{p} / \sqrt{3}$, while the latter predicts a frequency of $\omega_{s p}^{\prime}=\sqrt{\frac{2}{3}} \omega_{p}=\sqrt{2} \omega_{s p}$ $\approx 1.4 \omega_{s p}$. In comparison, the resonance according to the local theory is shifted to about $1.2 \omega_{s p}$ for the cavity case as shown in Figs. 4 and 5. The small discrepancy arises since damping is included in the dielectric function we used. Finally, shown in Figs. 6 and 7 are the emission properties (with the normalized decay rate in logarithmic values) as a function of the cavity size for diameters up to $10 \mathrm{~nm}$. Note
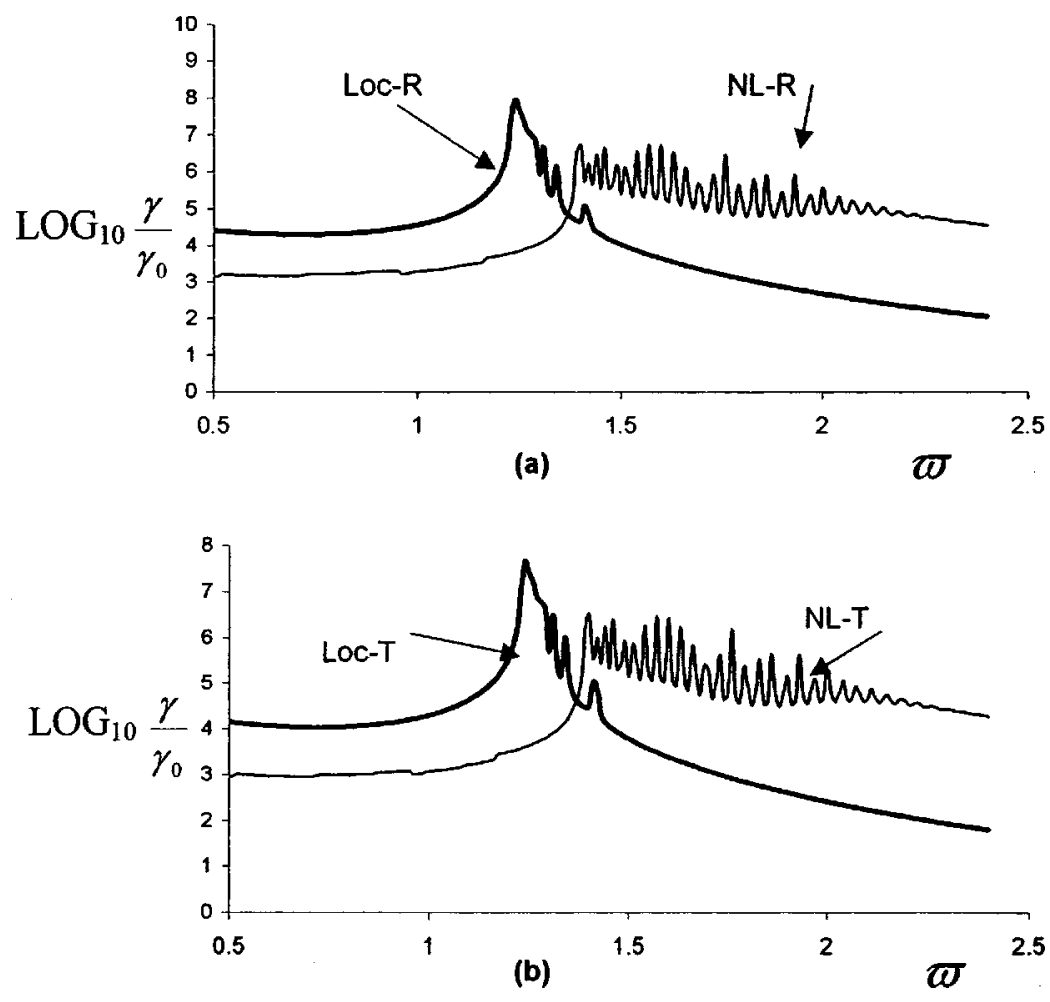

FIG. 4. Normalized decay rate as a function of normalized emission frequency for a fixed location of the molecule at $d=4.6 \mathrm{~nm}$ from the center of the $\mathrm{Ag}$ cavity of diameter equal to 10 $\mathrm{nm}$ for (a) a radial dipole and (b) a tangential dipole. Note that logarithmic values are used for the $y$ axis. 

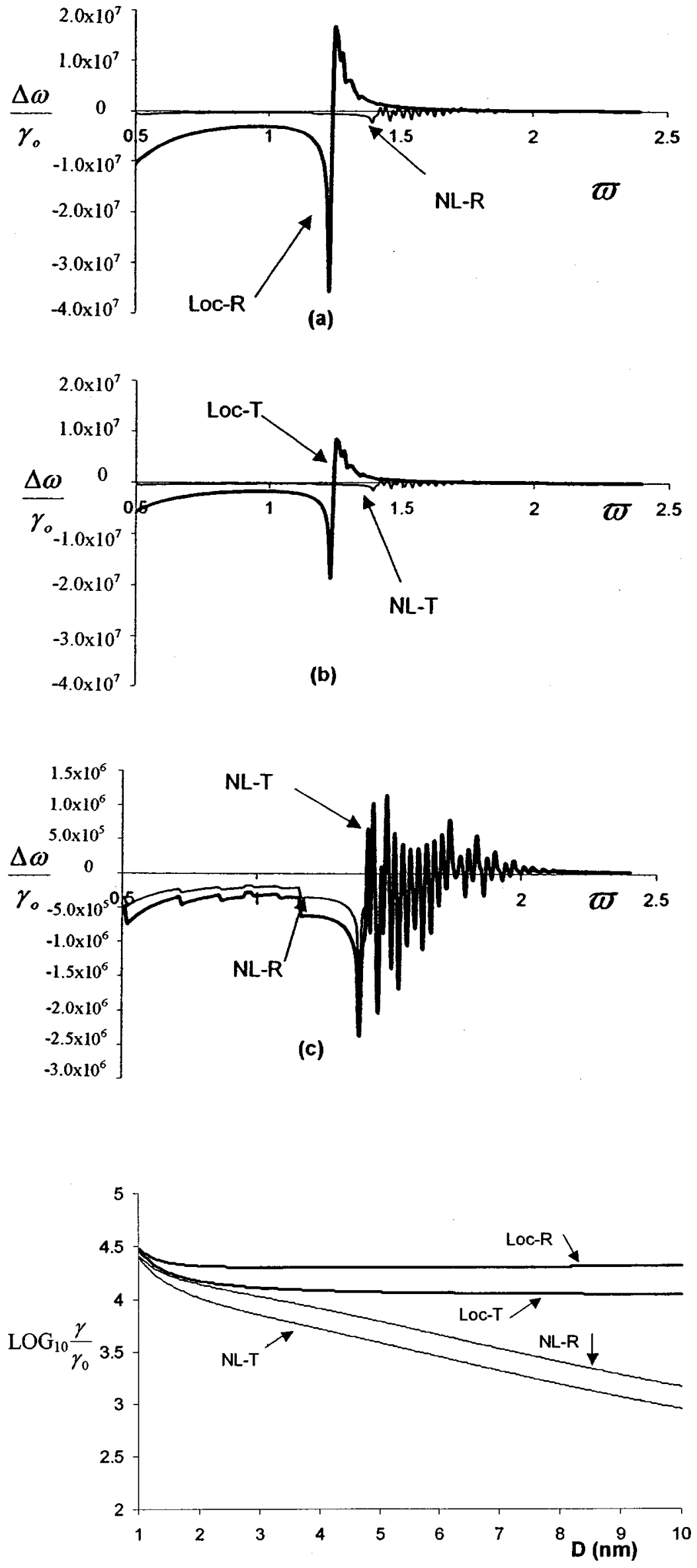

FIG. 5. Normalized frequency shift as a function of normalized emission frequency at $d$ $=4.6 \mathrm{~nm}$ from the center of a Ag cavity of diameter $=10 \mathrm{~nm}$ with results for (a) a radial dipole, (b) a tangential dipole, and (c) a comparison between the radial and tangential cases in the nonlocal theory.
FIG. 6. Normalized decay rate as a function of the diameter $(D)$ of the cavity with fixed emission frequency at $\varpi=0.7$ and fixed distance 0.4 $\mathrm{nm}$ from the cavity wall. Note that logarithmic values are used for the $y$ axis. 


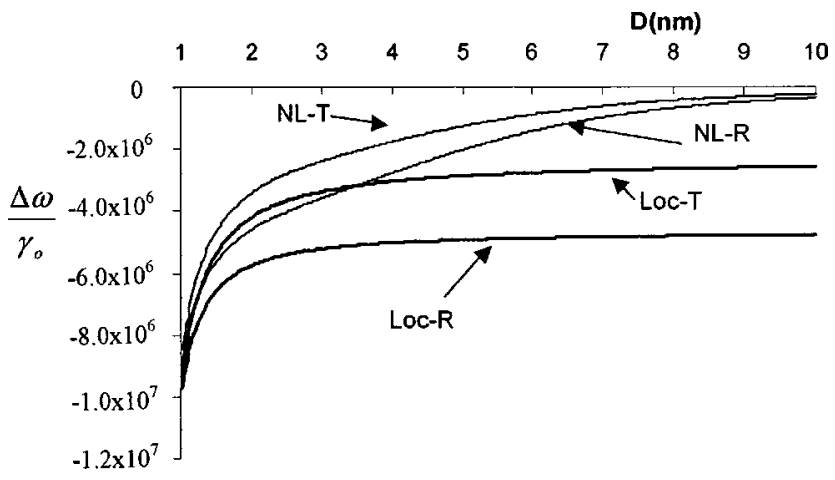

FIG. 7. Same as in Fig. 6, but results for the normalized frequency shift.

that here the molecule is fixed at a distance $(D / 2-d$ $=0.4 \mathrm{~nm}$ ) from the cavity wall. While it is seen that the surface-induced effects decrease with the increase in cavity size, in agreement with previous results established in the literature, ${ }^{14}$ one sees that the nonlocal effects once again lead to an overall decrease in such effects. Though this is in a direction leading to an account of the discrepancy observed between theory and experiment in the work of Barns et al., ${ }^{14}$ our current theory is not applicable to cavities with sizes much greater than the emission wavelengths as is the case in the work of Barns et al. ${ }^{14}$ Thus it would be of interest to extend the present nonlocal model to go beyond the longwavelength limit to account for all electrodynamic effects in the phenomena. Although retardation effects in the context of nonlocal electrodynamics have been studied before, ${ }^{24,25}$ most of these previous works are limited to either plane-wave incidence and/or plane-boundary geometry. To our knowledge, the same kind of effects for dipolar emissions in the vicinity of a spherical boundary have not yet been studied previously in the literature. ${ }^{26}$

\section{DISCUSSION AND CONCLUSION}

Within a simple model based on the theory of Fuchs and Claro for the multipolar polarizability of a sphere ${ }^{19}$ and the hydrodynamic model (HDM) for free electrons, we have illustrated the nonlocal effects on the interaction between a molecular dipole and a spherical cavity. It is now appropriate to give an assessment of the accuracy and the limitation of the results obtained from this simple model. First of all, the Fuchs-Claro theory is an application of the semiclassical infinite barrier (SCIB) model, which is known to be imperfect in several aspects, such as in the abrupt drop of the electronic density across the geometrical boundary. ${ }^{27}$ Recent work on the modification of the SCIB model to accommodate a smooth variation of this density across the surface ${ }^{28}$ can therefore provide one way of improving the accuracy of our present results. In addition, there also exist in the literature many different approaches going beyond the SCIB model which treat the surface nonlocal effects more accurately. ${ }^{27,29}$ Even within the SCIB model, one could have used more accurate nonlocal dielectric functions other than the HDM. It is well known that the HDM is accurate only for freeelectron metals like aluminum and will be limited for noble metals such as silver where the interband transitions from $s$ and $d$ electrons (and their mutual interaction) are important. In fact, there exist many models (e.g., the Lindhard-Mermin model) in which both plasmon and exciton can be accounted for and with respect to which the HDM is only the lowestorder approximation in the wave vector. ${ }^{30}$ In addition, Liebsch has established an approach based on the densityfunctional theory which can treat the $s$ - $d$ electronic interaction and lead to a more accurate description of the surface plasmon excitation for silver. ${ }^{31}$

Hence we see that there is plenty of room for one to improve on our present SCIB-HDM model which will lead to more accurate modeling results for the nonlocal effects. However, our intention here is to limit to a qualitative illustration of these effects using the simplest possible approach. In spite of this, we believe that our model should have some limited validity, even for noble metals, since it has been established experimentally that the free-electron model can account for the optical conductivity of these metals in the infrared and visible regions. ${ }^{32}$ In addition, there has been speculation that the errors due to application of the SCIB model may in some case cancel, fortuitously, with those from the use of the HDM when applied to silver, leading to a reasonable prediction of the dipolar surface plasmon shifts for silver particles as the particle radius decreases. ${ }^{27}$

Thus, in summary, the present results should have relevance to the fluorescence from molecules embedded in nanocavities. ${ }^{8,11,12}$ We also remark that our results can be easily extended to the case with "nanodroplets" by simply replacing $\xi_{l}$ by $\xi_{l}^{-1}$ in the expression for $\Delta_{l}^{\text {cav }}$ (with similar changes for the local results). For those experiments using liquid droplets of sizes in microns or tens of microns, our present model must be generalized to take into account electrodynamic retardation effects before they can be applied to such microcavities. However, the two general features we have observed with nonlocal effects- that is, (i) their significance for molecules near the cavity wall and (ii) the decrease in surface-induced effects-should have some general validity. In addition, we have observed that in the presence of nonlocal effects, the simple "reciprocity relation" between the results for the case of a sphere and those for a cavity no longer exists, and one must solve the two boundary value problems independently to obtain solutions for each of the two cases.

\section{ACKNOWLEDGMENTS}

Part of this work is based on the Ph.D. thesis of one of us (M.H.H.), who received financial support from Komatsu Silicon USA during his study in the graduate program at Portland State University. We also want to thank Professor J. Baudon for a correspondence on the results published in Ref. 18. Partial support from the Faculty Development Committee at Portland State University is also acknowledged. 
*Author to whom correspondence should be addressed.

${ }^{1}$ See, e.g., Cavity Quantum Electrodynamics, edited by P. Berman (Academic, San Diego, 1994).

${ }^{2}$ D. G. Deppe and C. Lei, J. Appl. Phys. 70, 3443 (1991).

${ }^{3}$ W. L. Barnes, J. Mod. Opt. 45, 661 (1998).

${ }^{4}$ M. Hopmeier, W. Guss, M. Deussen, E. O. Gobel, and R. F. Mahrt, Phys. Rev. Lett. 82, 4118 (1999).

${ }^{5}$ W. L. Barnes and P. Andrew, Nature (London) 400, 505 (1999).

${ }^{6}$ H. Chew, J. Chem. Phys. 87, 1355 (1987).

${ }^{7}$ Y. S. Kim, P. T. Leung, and T. F. George, Surf. Sci. 195, 1 (1988).

${ }^{8}$ V. V. Klimov, M. Ducloy, and V. S. Letokhov, J. Mod. Opt. 43, 549 (1996); V. V. Klimov and V. S. Letokhov, Chem. Phys. Lett. 301, 441 (1999).

${ }^{9}$ Y. Wu, Phys. Rev. A 61, 033803 (2000). This paper actually goes beyond the two-level model and considers a quasi-three-level system in the cavity.

${ }^{10}$ H. T. Dung, L. Knoll, and D.-G. Welsch, Phys. Rev. A 62, 053804 (2000).

${ }^{11}$ M. S. Tomas, Phys. Rev. A 63, 053811 (2001).

${ }^{12}$ A. Rahmani and G. W. Bryant, Phys. Rev. A 65, 033817 (2002). This work treats the cavity beyond the spherical shape via a numerical approach using the discrete dipole approximation

${ }^{13}$ H.-B. Lin, J. D. Eversole, C. D. Merritt, and A. J. Campillo, Phys. Rev. A 45, 6756 (1992).

${ }^{14}$ M. D. Barnes, C. Y. Kung, W. B. Whitten, J. M. Ramsey, S. Arnold, and S. Holler, Phys. Rev. Lett. 76, 3931 (1996).

${ }^{15}$ M. D. Barnes, N. Lermer, C. Y. Kung, W. B. Whitten, J. M. Ramsey, and S. C. Hill, Opt. Lett. 22, 1265 (1997).

${ }^{16}$ M. V. Badilevsky and D. F. Parsons, J. Chem. Phys. 105, 3734 (1996).

${ }^{17}$ R. R. Chance, A. Prock, and R. Silbey, Adv. Chem. Phys. 37, 1 (1978).

${ }^{18}$ B. Labani, C. Girard, and S. Maghezzi, Mol. Phys. 69, 85 (1990);
B. Labani, M. Boustimi, and J. Baudon, Phys. Rev. B 55, 4745 (1997); M. Boustimi, J. Baudon, A. Semlali, R. Mathevet, and B. Labani, Surf. Sci. 437, 91 (1999); M. Boustimi, J. Baudon, J. Robert, A. Semlali, and B. Labani, Phys. Rev. B 62, 7593 (2000).

${ }^{19}$ R. Fuchs and F. Claro, Phys. Rev. B 35, 3722 (1987).

${ }^{20}$ P. T. Leung, Phys. Rev. B 42, 7622 (1990); P. T. Leung and M. H. Hider, J. Chem. Phys. 98, 5019 (1993).

${ }^{21}$ Also noted is that the results obtained for the dipole-cavity problem are not consistent between the 1997 and 1999 papers in Ref. 18. We believe that the one given in the 1999 paper is correct.

${ }^{22}$ S. C. Hill, G. Videen, and J. D. Pendleton, J. Opt. Soc. Am. B 14, 2522 (1997).

${ }^{23}$ See, e.g., J. D. Jackson, Classical Electrodynamics (Wiley, New York, 1999), p. 158.

${ }^{24}$ W. H. Weber and G. W. Ford, Phys. Rev. Lett. 44, 1774 (1980).

${ }^{25}$ D. R. Penn and R. W. Rendell, Phys. Rev. Lett. 47, 1067 (1981); Phys. Rev. B 26, 3047 (1982).

${ }^{26}$ Although these effects have been included for a spherical boundary in the case of local electrodynamics previously in the literature: see, e.g., R. Ruppin, J. Chem. Phys. 76, 1681 (1982) and Ref. 7.

${ }^{27}$ Spatial Dispersion in Solids and Plasmas edited by P. Halevi (North-Holland, Amsterdam, 1992).

${ }^{28}$ A. T. George, Opt. Commun. 188, 321 (2001).

${ }^{29}$ See also P. J. Feibelman, Prog. Surf. Sci. 12, 287 (1982); K. Kempa and W. L. Schaich, Phys. Rev. B 39, 13139 (1989).

${ }^{30}$ See, e.g., G. D. Mahan, Many-Particle Physics, 2nd ed. (Plenum, New York, 1990). See also the references given in R. Chang, P. T. Leung, S. H. Lin, and W. S. Tse, Phys. Rev. B 62, 5168 (2000).

${ }^{31}$ A. Liebsch, Phys. Rev. Lett. 71, 145 (1993); Phys. Rev. B 48, 11317 (1993).

${ }^{32}$ R. T. Beach and R. W. Christy, Phys. Rev. B 16, 5277 (1977). 